\title{
Flexible Recommendations in CourseRank
}

\author{
Hector Garcia-Molina
}

\begin{abstract}
CourseRank is a course planning tool we have developed for Stanford students, and is already in use by most undergraduates. For CourseRank, we have developed a "flexible recommendations" engine for defining recommendation strategies as high level workflows. By selecting a workflow and providing parameters (e.g., a filter condition for biology classes), students can receive personalized recommendations that better suit their needs. In this talk I will give an overview of CourseRank and its recommendation engine.
\end{abstract}

\section{Speaker Bio}

Hector Garcia-Molina is the Leonard Bosack and Sandra Lerner Professor in the Departments of Computer Science and Electrical Engineering at Stanford University, Stanford, California. He was the chairman of the Computer Science Department from January 2001 to December 2004. From 1997 to 2001 he was a member the President's Information Technology Advisory Committee (PITAC). From August 1994 to December 1997 he was the Director of the Computer Systems Laboratory at Stanford. From 1979 to 1991 he was on the faculty of the Computer Science Department at Princeton University, Princeton, New Jersey. His research interests include distributed computing systems, digital libraries and database systems. He received a BS in electrical engineering from the Instituto Tecnologico de Monterrey, Mexico, in 1974. From Stanford University, Stanford, California, he received in 1975 a MS in electrical engineering and a $\mathrm{PhD}$ in computer science in 1979. He holds an honorary $\mathrm{PhD}$ from ETH Zurich (2007). Garcia-Molina is a Fellow of the Association for Computing Machinery and of the American Academy of Arts and Sciences; is a member of the National Academy of Engineering; received the 1999 ACM SIGMOD Innovations Award; is on the Technical Advisory Board of DoCoMo Labs USA, Yahoo Search \& Marketplace; is a Venture Advisor for Diamondhead Ventures, and is a member of the Board of Directors of Oracle and Kintera. 